\title{
西达本胺衍生物的设计合成及抗肿瘤活性评价
}



(山东大学药学院化学生物学教育部重点实验室 济南 250012)

\begin{abstract}
摘要 以西达本胺为基础设计合成了一系列新型组蛋白去乙酰化酶(HDACs)抑制剂, 以提高与 $\mathrm{Zn}^{2+}$ 的鳌合作用和亚型 选择性. 大部分化合物表现出一定的抗肿瘤增殖活性. 其中, $(E)-N-(4$-氨基-6-氟-[1,1'联苯]3-基)-4-((3-(吡啶-3-基)丙烯 酰氨基)甲基)苯甲酰胺(7i) 和 $(E)$ - $N$-(2-氨基-4-氟-5-(噻吩-2-基)苯基)-4-((3-(吡啶-3-基)丙烯酰胺基)甲基)苯甲酰胺(7j)抗 肿瘤增殖活性最佳, 对 Jurkat 细胞的 $\mathrm{IC}_{50}$ 分别为 3.29 和 $12.59 \mu \mathrm{mol} / \mathrm{L}$, 并且这两个化合物表现出一定的 HDAC 抑制活 性, 为更有潜力的西达本胺衍生物的设计合成提供了新思路.
\end{abstract}

关键词＼cjkstart西达本胺; HDACs; 抗肿瘤活性; 苯甲酰胺

\section{Design, Synthesis and Evaluation of Anti-tumor Activities of Chidamide Derivatives}

\author{
Zhang, Xiangna $^{\dagger} \quad$ He, Feng ${ }^{\dagger} \quad$ Zhang, Qiuqiong Lü, Jiahui Xu, A'na \\ Yu, Chenggong Qu, Ying Wu, Jingde* \\ (Key Laboratory of Chemical Biology (Ministry of Education), School of Pharmaceutical Science, \\ Shandong University, Jinan 250012)
}

\begin{abstract}
A series of novel chidamide based histone deacetylases (HDACs) inhibitors were rationally designed and synthesized to increase the $\mathrm{Zn}^{2+}$ chelating and selectivity. Biological characterization established that most of the compounds showed moderate antiproliferative activitites in cancer cell lines. Among the tested analogs, (E)- $N-(4-a m i n o-6-$ fluoro-[1,1'-biphenyl]-3-yl)-4-((3-(pyridin-3-yl)acrylamido)methyl)benzamide (7i) and $(E)-N$-(2-amino-4-fluoro-5-(thiophen2-yl)phenyl)-4-((3-(pyridin-3-yl)acrylamido)methyl)benzamide (7j) exhibit the most potent antiproliferative activity with $\mathrm{IC}_{50}$ of 3.29 and $2.59 \mu \mathrm{mol} / \mathrm{L}$ in Jurkat cells, respectively. Furthermore, these two compounds have a certain HDAC inhibitory activity. Collectively, the results partly encourage further development of more potential analogs based on chidamide.

Keywords chidamide; HDACs; anti-tumor activity; benzamide
\end{abstract}

\section{Introduction}

With the advances in molecular genetics and genomics studies, many promising biological targets related to tumor pathogenesis have been identified. Histone deacetylases (HDACs) have been extensively studied to develop effective antitumor therapeutics in recent years. Nucleosomes are the core of histone and include a spherical c-terminal structure and an amino acid residue $n$-termina. ${ }^{[1]}$ Histone modification mainly includes acetylation and deacetylation, methylation and demethylation, phosphorylation and dephosphorylation, ubiquitination and deubiquitination, among which the acetylation and deacetylation modify the chromatin morphology by regulating the assembly of nucleosomes. ${ }^{[2]}$ Functionally, HDACs remove acetyl moiety on 1-amino of $\mathrm{N}$-terminal lysine residues by hydrolysis, thus increasing the electropositivity of the lysine residues. As a result, it binds tightly to electronegative DNA and hinders transcription factors, RNA polymerases and other transcription-regulatory proteins from binding to DNA, leading to gene transcription inhibition. ${ }^{[3,4]}$ To date, the HDACs can be classified into four subgroups and 18 subtypes. Accumulating evidences have demonstrated the

\footnotetext{
* Corresponding author. E-mail: wujingde70@sdu.edu.cn

Received December 19, 2018; revised January 12, 2019; published online March 29, 2019.

Project supported by the Key Research and Development Plan of Shandong Province (No. 2017CXGC1401)

山东省重点研发计划(No. 2017CXGC1401)资助项目.

共同第一作者(These authors contributed equally to this work).
} 
pathological roles of the HDACs in many human diseases, such as cancer, inflammation, and neurodegenerative disorders. Cellular localization varies for different subtypes of HDACs, therefore, development of subtype selective HDACs inhibitors represents a strategy to develop disease specific therapeutics. ${ }^{[5,6]}$

Chidamide has been approved as a subtype-selective histone deacetylase inhibitor for relapsed or refractory peripheral T-cell lymphoma. ${ }^{[7]}$ Chidamide provides specific inhibition of class I HDACs that are associated with tumor genesis and are related to chromatin remodeling and gene transcriptional regulation (i.e., epigenetic regulation) (Figure 1). The compound affects the cell cycle of lymphatic, hematological tumors and induces apoptosis. Furthermore, it enhances the activity of natural killer (NK) and antigen-specific cytotoxic T-cells (CTL) to inhibit the inflammatory response of tumor tissue. ${ }^{[8]}$ However, adverse reactions have been observed for chidamide, especially in hematology and gastrointestinal tract. ${ }^{[9,10]}$ Therefore, structural optimization of chidamide may provide novel analogs with reduced toxicity and improved potency.

The side effects of chidamide are mainly caused by the low selectivity within HDACs subtype. The structure-activity relationships have provided valuable information on the binding interactions with the HDACs (Fig- ure 1). ${ }^{[11]}$

Studies of the benzamide-based HDACs inhibitors, as exemplified by MS275 and MGCD-0103, revealed that only HDAC1 and HDAC2 have a deep internal cavity in the bottom of $\mathrm{Zn}^{2+}$ chelating area. So introduction of aromatic ring or aromatic heterocyclic in the para-position of amino in B ring significantly improved the potency and selectivity against class I HDACs. ${ }^{[9]}$ Based on these observations, we designed a series of chidamide derivatives to probe the influence of substitution on the analine moiety of Chidamide (Figure 2).

\section{Results and discussion}

\subsection{Chemistry}

The synthesis of compound 7 was shown in Scheme 1. Briefly, intermediate 2a was obtained by the bromination of $\mathbf{1}$ in the para-position of the amine with $\mathrm{N}$-bromo-succinimide (NBS) and azobisisobutyronitrile, azodiisobutyronitrile $(\mathrm{AIBN}) .{ }^{[12]}$ Boc protection yielded 3a, which was reduced with $\mathrm{Zn}$ powder to give $4 \mathbf{a} .^{[13,14]}$ Condensation of $4 \mathbf{a}$ with 5 afforded 6 . Finally, reaction of $6 \mathbf{a}$ with various aromatic borates under Suzuki reaction conditions provided the target compounds.<smiles>Nc1ccccc1NC(=O)c1ccc(CNC(=O)/C=C/c2cccnc2)cc1</smiles>
Chidamide

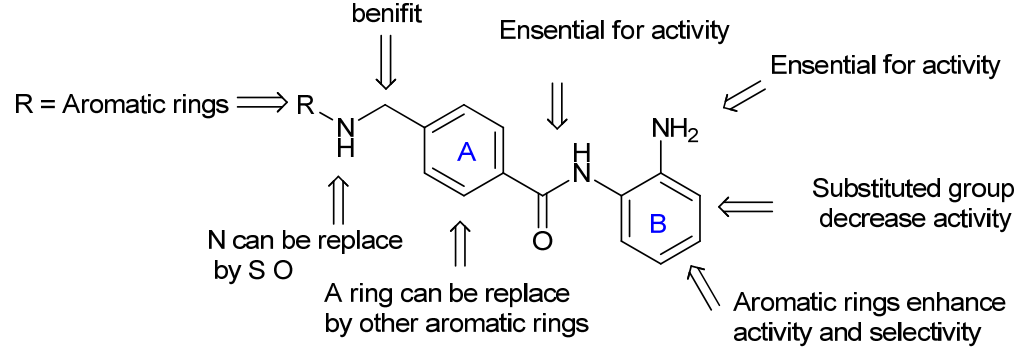

Figure 1 Chidamide and the structure-activity relationship of benzamide class HDACs inhibitors<smiles>Nc1cc(F)ccc1NC(=O)c1ccc(CNC(=O)/C=C/c2cccnc2)cc1</smiles><smiles>[R]c1ccc(N)c(NC(=O)c2ccc(CNC(=O)OCc3cccnc3)cc2)c1</smiles><smiles>[R]O[R16](=O)c1ccc(CNc2nccc(-c3cccnc3)n2)cc1</smiles><smiles>[R]Oc1cc([R])c(NC(=O)c2ccc(CNC(=O)/C=C/c3cccnc3)cc2)cc1N</smiles><smiles>[R]=CC(C)CCCC(C)c1ccc(CC(C)c2ccc(C)cc2)cc1</smiles>

$\mathrm{R}^{1}=\hat{s}$<smiles>[R]=C(C)c1ccncc1</smiles>

Figure 2 Design of the chidamide derivatives 


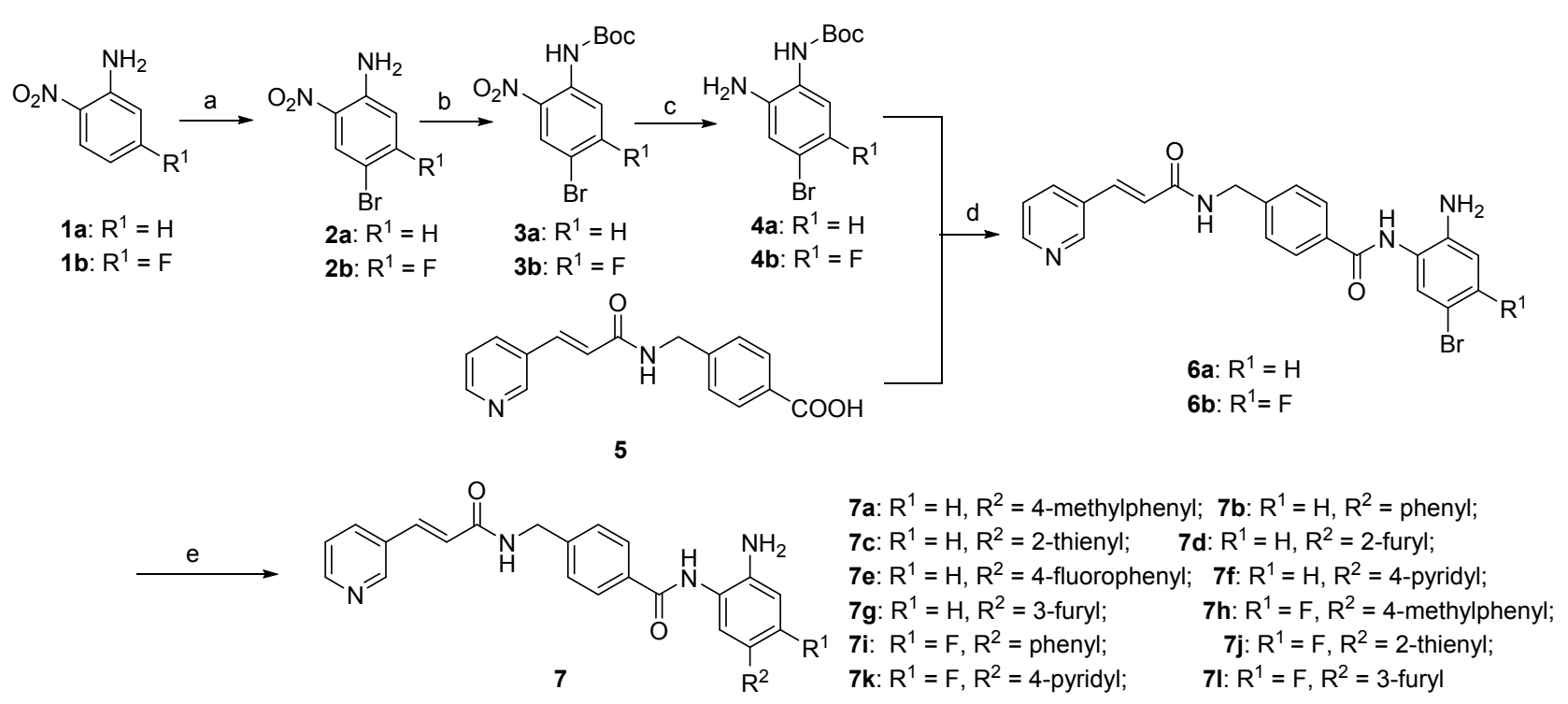

Reagents and conditions: (a) NBS, $\mathrm{AcOH}, 100{ }^{\circ} \mathrm{C}, 2 \mathrm{~h}$; (b) (Boc) $2 \mathrm{O}$, TEA, DMAP, DCM, r.t., 2 h; (c) $\mathrm{Zn}, \mathrm{NH}_{4} \mathrm{Cl}, \mathrm{H}_{2} \mathrm{O}, \mathrm{MeOH}, 80^{\circ} \mathrm{C}, 2 \mathrm{~h}$; (d) (i) TBTU, TEA, THF, $70{ }^{\circ} \mathrm{C}, 3 \mathrm{~h}$; (ii) $\mathrm{HCl}$, EtOAc, r.t., $1 \mathrm{~h}$; (e) corresponding boronic acid derivatives, $\mathrm{Pd}\left(\mathrm{Ph}_{3} \mathrm{P}\right)_{4}$, toluene, $\mathrm{EtOH}, \mathrm{K}_{2} \mathrm{CO}_{3}, \mathrm{NaF}, \mathrm{H}_{2} \mathrm{O}$, Ar, $100{ }^{\circ} \mathrm{C}, 5 \mathrm{~h}$.

Scheme 1 Synthesis of the chidamide derivatives

\subsection{In vitro HDACs enzyme inhibition.}

HeLa nuclear extracts (mainly including HDAC1 and HDAC2) were employed to evaluate the inhibition of these analogs on enzymatic activity. chidamide and MS275 were used as positive controls. As shown in Table 1, the only analogs $7 \mathbf{h}$ and $7 \mathbf{i}$ showed moderate inhibitory activity with $\mathrm{IC}_{50}$ of 13.46 and $18.82 \mu \mathrm{mol} / \mathrm{L}$, respectively while the other analogs showed no inhibition. The results suggest that fluorine atom is essential to the activity of chidamide and aromatic derivation at the para-position of amino moiety does not improve the inhibitory activity.

\subsection{Evaluation of anti-proliferation activity.}

Three tumor cell lines, K562, Jurkat and HeLa, were selected to conduct a preliminary evaluation of the anti-proliferative activities for the compounds at $25 \mu \mathrm{mol} / \mathrm{L}$. As shown in Table 2, surprisingly, all the tested compounds showed comparable antiproliferative activities as Chidamide, although most of them did not show inhibitory activity in the enzymatic assay. Compounds $\mathbf{7 h}$ and $\mathbf{7} \mathbf{i}$, the ones that showed moderate anti-HDACs enzyme activity, exhibited improved inhibition on the proliferation of tested cancer cell lines when compared to Chidamide. Further characterization established the $\mathrm{IC}_{50}$ of 5.28 and 3.29 $\mu \mathrm{mol} / \mathrm{L}$ for $\mathbf{7 h}$ and $\mathbf{7 i}$, respectively.

\section{Conclusions}

A series of Chidamide derivatives have been designed and synthesized to understand the SAR of benzamide based class I HDACs inhibitors. Our studies demonstrated that derivatization on the para-position of the $\mathrm{B}$ ring does not improve its HDACs inhibitory activity. It may hint that the para-position of amino moiety does not occupy the inner cavity and this may guide the design of next genera- tion of benzamide based HDACs inhibitors.

\section{Experimental section}

\subsection{Chemistry}

All commercially available starting materials reagents and solvents were used without further purification unless otherwise stated. All reactions were monitored by TLC with $0.25 \mathrm{~mm}$ silica gel plates (60GF-254). UV light and ferric chloride were used to visualize the spots. Silica gel was used for column chromatography purification. ${ }^{1} \mathrm{H}$ NMR and ${ }^{13} \mathrm{C}$ NMR spectra were obtained on a Bruker DRX spectrometer at $400 \mathrm{MHz}$. High resolution mass spectra were conducted by Shandong Analysis and Test Centre in Jinan, China. Melting points were determined on an uncorrected electrothermal melting point apparatus.

\subsubsection{General produce for intermediate $\mathbf{2} \sim \mathbf{4}$}

4-Bromo-2-nitroaniline (2a) ${ }^{[12]}$ NBS (1.29 g, 7.24 mmol) was added to a solution of $1 \mathrm{a}(1 \mathrm{~g}, 7.24 \mathrm{mmol})$ in $15 \mathrm{~mL}$ glacial acetic acid. The reaction was stirred at $100{ }^{\circ} \mathrm{C}$ for $2 \mathrm{~h}$. After cooling to room temperature, $50 \mathrm{~mL}$ of water was added and under ultraphonic for $5 \mathrm{~min}$. The mixture was filtered, dried over anhydrous sodium sulfate and 4-bromo-2-nitroaniline (2a) was obtained as yellow cold $\left(1.29 \mathrm{~g}, 82 \%\right.$ yield). ${ }^{1} \mathrm{H}$ NMR $\left(400 \mathrm{MHz}, \mathrm{CDCl}_{3}\right) \delta$ : 8.25 (s, $J=2.4 \mathrm{~Hz}, 1 \mathrm{H}), 7.41$ (dd, $J=8.8,2.3 \mathrm{~Hz}, 1 \mathrm{H})$, $6.72(\mathrm{~d}, J=8.8 \mathrm{~Hz}, 1 \mathrm{H}), 6.10(\mathrm{~s}, 2 \mathrm{H})$

5-Bromo-5-fluoro-2-nitroaniline (2b): ${ }^{[15]}$ The synthesis is the same as intermediate 2a (76\% yield). ${ }^{1} \mathrm{H}$ NMR (400 MHz, DMSO- $\left.d_{6}\right) \delta: 8.26(\mathrm{~d}, J=7.4 \mathrm{~Hz}, 1 \mathrm{H}), 7.71(\mathrm{~s}, 2 \mathrm{H})$, $6.94(\mathrm{~d}, J=11.0 \mathrm{~Hz}, 1 \mathrm{H})$.

tert-Butyl (4-bromo-2-nitrophenyl)carbamate (3a): TEA $(1.71 \mathrm{~g}, 16.95 \mathrm{mmol})$, DMAP $(0.28 \mathrm{~g}, 2.26 \mathrm{mmol})$ 
Table 1 HDACs inhibition activity of chidamide derivatives<smiles>[R]c1cc(N)c(NC(=O)c2ccc(CNC(=O)/C=C/c3cccnc3)cc2)cc1[R]</smiles>

\begin{tabular}{|c|c|c|c|c|c|c|c|}
\hline Compd. & $\mathrm{R}^{1}$ & $\mathrm{R}^{2}$ & $\mathrm{IC}_{50}{ }^{a} /\left(\mu \mathrm{mol} \cdot \mathrm{L}^{-1}\right)$ & Compd. & $\mathrm{R}^{1}$ & $\mathrm{R}^{2}$ & $\mathrm{IC}_{50}{ }^{a} /\left(\mu \mathrm{mol} \cdot \mathrm{L}^{-1}\right)$ \\
\hline $7 a$ & & & $>50$ & $7 \mathrm{~g}$ & & & $>50$ \\
\hline $7 b$ & & & $>50$ & $7 \mathrm{~h}$ & & & 13.46 \\
\hline $7 \mathrm{c}$ & & & $>50$ & $7 \mathbf{i}$ & & & 18.82 \\
\hline $7 d$ & & & $>50$ & $7 \mathbf{j}$ & & & $>50$ \\
\hline $7 e$ & & & $>50$ & $7 \mathbf{k}$ & & & $>50$ \\
\hline $7 f$ & & & $>50$ & 71 & 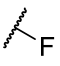 & & $>50$ \\
\hline Chidamide & $\alpha_{F}$ & & 2.89 & MS275 & - & - & 1.72 \\
\hline
\end{tabular}

${ }^{a}$ Assay were performed in replicate $(n \geqslant 2)$, the SD values are $<20 \%$ of the mean.

Table 2 Preliminary screening of anti-proliferative activity of chidamide derivatives on four tumor cells ${ }^{a}$

\begin{tabular}{|c|c|c|c|c|c|c|c|}
\hline \multirow{2}{*}{ Compd. } & \multicolumn{3}{|c|}{ Antiproliferative activity $(\%)$ at $25 \mu \mathrm{mol} / \mathrm{L}$} & \multirow{2}{*}{ Compd. } & \multicolumn{3}{|c|}{ Antiproliferative activity $(\%)$ at $25 \mu \mathrm{mol} / \mathrm{L}$} \\
\hline & K562 & Jurkat & $\mathrm{HeLa}$ & & K562 & Jurkat & $\mathrm{HeLa}$ \\
\hline $7 \mathbf{a}$ & 56.20 & 47.48 & 43.22 & $7 \mathrm{~g}$ & 43.51 & 69.12 & 34.40 \\
\hline $7 \mathbf{b}$ & 61.76 & 38.96 & 49.45 & $7 \mathbf{h}$ & 71.16 & 86.43 & 39.27 \\
\hline $7 c$ & 50.48 & 34.16 & 47.36 & $7 \mathbf{i}$ & 83.45 & 89.01 & 45.13 \\
\hline $7 d$ & 29.86 & 52.24 & 28.48 & $7 \mathbf{j}$ & 47.22 & 65.93 & 40.80 \\
\hline $7 e$ & 58.02 & 80.55 & 37.86 & $7 \mathbf{k}$ & 73.49 & 72.78 & 32.73 \\
\hline $7 f$ & 42.21 & 65.79 & 41.00 & 71 & 79.65 & 67.00 & 39.72 \\
\hline Chidamide & 65.20 & 78.96 & 55.76 & & & & \\
\hline
\end{tabular}

${ }^{a}$ Assay were performed in replicate $(n \geqslant 2)$, the SD values are $<20 \%$ of the mean.

Table 3 Anti-proliferation $\mathrm{IC}_{50}$ values $\left(\mu \mathrm{mol} \cdot \mathrm{L}^{-1}\right)$ of derivatives $7 \mathbf{h} \sim \mathbf{7 l}$ and chidamide against $\mathrm{K} 562$ and Jurkat cell lines ${ }^{a}$

\begin{tabular}{lcccccc}
\hline Compd. & $\mathbf{7 h}$ & $\mathbf{7 i}$ & $\mathbf{7 j}$ & $\mathbf{7 k}$ & $\mathbf{7 1}$ \\
\hline K562 & 22.18 & 32.54 & 18.70 & 20.67 & 30.10 \\
Jurkat & 5.28 & 3.29 & 2.59 & 12.78 & 6.72 \\
\hline
\end{tabular}

${ }^{a}$ Assay were performed in replicate $(n \geqslant 2)$, the SD values are $<20 \%$ of the mean.

were added to the solution of $\mathbf{2 a}(2.45 \mathrm{~g}, 11.3 \mathrm{mmol})$ in $\mathrm{CH}_{2} \mathrm{Cl}_{2}(30 \mathrm{~mL})$ under the ice bath. Then (Boc) $)_{2} \mathrm{O}(2.45 \mathrm{~g}$, $11.30 \mathrm{mmol}$ ) was added and the reaction was stirred for 30 $\min$ at $0{ }^{\circ} \mathrm{C}$. The reaction was wash with water $(30$ $\mathrm{mL} \times 2)$ and brine $(30 \mathrm{~mL})$. The organic phase was dried with anhydrous magnesium sulfate for $30 \mathrm{~min}$, filtered and the solvent removed under reduced pressure. tert-Butyl (4-bromo-2-nitrophenyl)carbamate (3a) was obtained without purification as yellow solid $\left(2.13 \mathrm{~g}, 59 \%\right.$ yield). ${ }^{1} \mathrm{H}$ NMR (400 MHz, DMSO- $\left.d_{6}\right) \delta: 9.68(\mathrm{~s}, 1 \mathrm{H}), 8.13$ (d, $J=$
$2.3 \mathrm{~Hz}, 1 \mathrm{H}), 7.87$ (dd, $J=8.8,2.4 \mathrm{~Hz}, 1 \mathrm{H}), 7.60$ (d, $J=8.8$ $\mathrm{Hz}, 1 \mathrm{H}), 1.45$ (s, 9H).

tert-Butyl (4-bromo-5-fluoro-2-nitrophenyl)carbamate (3b): The synthesis is the same as intermediate $\mathbf{2 a}$ and the product was yellow solid (79\% yield). m.p. 100 $103{ }^{\circ} \mathrm{C}$; ${ }^{1} \mathrm{H}$ NMR (600 MHz, DMSO-d $) \delta: 9.78$ (s, 1H), 8.39 (d, $J=7.0 \mathrm{~Hz}, 1 \mathrm{H}), 7.79(\mathrm{~d}, J=10.5 \mathrm{~Hz}, 1 \mathrm{H}), 1.47(\mathrm{~s}, 9 \mathrm{H}) ;{ }^{13} \mathrm{C}$ NMR (151 MHz, DMSO) $\delta: 161.24(\mathrm{~d}, J=252.1 \mathrm{~Hz})$, $152.30,137.40,135.28$ (d, $J=11.9 \mathrm{~Hz}), 130.96$ (d, $J=3.0$ Hz), 110.68 (d, $J=28.5 \mathrm{~Hz}), 102.05$ (d, $J=23.5 \mathrm{~Hz})$, 


\section{$81.92,28.26$.}

tert-Butyl(2-amino-4-bromophenyl)carbamate $\quad \mathbf{( 4 a )})^{[14]}$ The intermediate $3 \mathbf{a}(1 \mathrm{~g}, 3.15 \mathrm{mmol})$ was dissolved in 20 $\mathrm{mL}$ of methanol and $10 \mathrm{~mL}$ of sat. aq. $\mathrm{NH}_{4} \mathrm{Cl}$, then zinc powder (1.02 g, $15.75 \mathrm{mmol})$ was added. The reaction was stirred at $80{ }^{\circ} \mathrm{C}$ for $30 \mathrm{~min}$ and then kept at room temperature for $2 \mathrm{~h}$. Zinc powder was filtered out by diatomite and methanol was removed under reduced pressure. Then 10 $\mathrm{mL}$ of water was added. The reaction was extracted with ethylacetate $(20 \mathrm{~mL} \times 3)$ and the organic phase was dried with anhydrous magnesium sulfate. Then the solvent was removed under reduced pressure to provide pale yellow solid (0.89 g, 98\% yield). ${ }^{1} \mathrm{H}$ NMR (400 MHz, DMSO- $d_{6}$ ) $\delta: 8.30(\mathrm{~s}, 1 \mathrm{H}), 7.16(\mathrm{~d}, J=8.4 \mathrm{~Hz}, 1 \mathrm{H}), 6.85(\mathrm{~d}, J=2.3$ $\mathrm{Hz}, 1 \mathrm{H}), 6.64$ (dd, $J=8.5,2.3 \mathrm{~Hz}, 1 \mathrm{H}), 5.14$ (s, 2H), 1.45 (s, 9H).

tert-Butyl (2-amino-4-bromo-5-fluorophenyl)carbamate (4b): The synthesis is the same as intermediate (4a). Pale yellow solid (96\% yield). m.p. $114 \sim 118{ }^{\circ} \mathrm{C}$; ${ }^{1} \mathrm{H}$ NMR $\left(600 \mathrm{MHz}, \mathrm{DMSO}-d_{6}\right) \delta: 8.50(\mathrm{~s}, 1 \mathrm{H}), 7.39(\mathrm{~d}, J=10.9 \mathrm{~Hz}$, $1 \mathrm{H}), 6.93(\mathrm{~d}, J=7.1 \mathrm{~Hz}, 1 \mathrm{H}), 5.05$ (s, 2H), 1.47 (s, 9H); ${ }^{13} \mathrm{C}$ NMR (151 MHz, DMSO- $\left.d_{6}\right) \delta: 153.54,150.11(\mathrm{~d}, J=$ $231.2 \mathrm{~Hz}), 138.28,124.87$ (d, $J=9.0 \mathrm{~Hz}), 118.24,110.86$, 101.82 (d, $J=22.8 \mathrm{~Hz}$ ), 80.02, 28.54; HRMS (ESI) calcd for $\mathrm{C}_{11} \mathrm{H}_{15} \mathrm{BrFN}_{2} \mathrm{O}_{2}\left([\mathrm{M}+\mathrm{H}]^{+}\right)$305.0295, found 305.0299.

(E)-N-(2-Amino-5-bromophenyl)-4-((3-(pyridin-3-yl)acrylamido)methyl)benzamide (6a): Triethylamine (TEA) (2.09 g, $20.73 \mathrm{mmol}$ ) and $O$-(benzotriazol-1-yl)- $N, N, N^{\prime}, N^{\prime}-$ tetramethyluronium tetrafluoroborate (TBTU) (4.44 g, $13.82 \mathrm{mmol}$ ) were added to a solution of intermediate $\mathbf{5}$ in methylene chloride and were stirred for $1 \mathrm{~h}$ at room temperature. Then TEA (2.09 g, $20.73 \mathrm{mmol})$ and intermediate 4a (4.00 g, $13.82 \mathrm{mmol}$ ) were added. The mixture was stirred at room temperature for $2 \mathrm{~h}$. The organic phase was washed with water, brine and dried with anhydrous magnesium sulfate. The solvent was removed under reduced pressure. The crude product was purified by Flash chromatography (FC) as a grey solid. The product was added to sat. aq. HCl-ethyl acetate and stirred at room temperature for $1 \mathrm{~h}$. The mixture was filtered to give a white solid. Added the solid in water and tuned to alkaline $(\mathrm{pH}=8 \sim$ $9)$. The solvent was filtered and $(E)-N$-(2-amino-5-bromophenyl)-4-((3-(pyridin-3-yl)acrylamido) methyl)benzamide (6a) was obtained. $1.75 \mathrm{~g}$, yield 29\%. ${ }^{1} \mathrm{H}$ NMR (400 MHz, DMSO- $\left.d_{6}\right) \delta: 9.65(\mathrm{~s}, 1 \mathrm{H}), 8.78(\mathrm{~d}, J=2.0 \mathrm{~Hz}, 2 \mathrm{H}), 8.56$ $(\mathrm{dd}, J=4.7,1.3 \mathrm{~Hz}, 1 \mathrm{H}), 8.01(\mathrm{~d}, J=8.0 \mathrm{~Hz}, 1 \mathrm{H}), 7.95$ (d, $J=8.2 \mathrm{~Hz}, 2 \mathrm{H}), 7.53(\mathrm{~d}, J=15.9 \mathrm{~Hz}, 1 \mathrm{H}), 7.48 \sim 7.40(\mathrm{~m}$, $3 \mathrm{H}), 7.39(\mathrm{~d}, J=2.2 \mathrm{~Hz}, 1 \mathrm{H}), 7.11(\mathrm{dd}, J=8.6,2.3 \mathrm{~Hz}$, $1 \mathrm{H}), 6.83(\mathrm{~d}, J=15.9 \mathrm{~Hz}, 1 \mathrm{H}), 6.74(\mathrm{~d}, J=8.6 \mathrm{~Hz}, 1 \mathrm{H})$, $5.14(\mathrm{~s}, 2 \mathrm{H}), 4.50(\mathrm{~d}, J=5.9 \mathrm{~Hz}, 2 \mathrm{H})$.

(E)- $N$-(2-Amino-5-bromo-4-fluorophenyl)-4-((3-(pyridin-3-yl)acrylamido)methyl)benzamide (6b): The synthesis is the same as 6a. Grey solid and yield 42\%. m.p. 117 $120{ }^{\circ} \mathrm{C} ;{ }^{1} \mathrm{H}$ NMR (600 MHz, DMSO- $\left.d_{6}\right) \delta: 9.69$ (s, $\left.1 \mathrm{H}\right)$, 8.85 (t, $J=6.0 \mathrm{~Hz}, 1 \mathrm{H}), 8.78$ (d, $J=2.3 \mathrm{~Hz}, 1 \mathrm{H}), 8.56$ (d, $J=4.7 \mathrm{~Hz}, 1 \mathrm{H}), 8.02(\mathrm{dt}, J=8.0,2.0 \mathrm{~Hz}, 2 \mathrm{H}), 7.96(\mathrm{~d}, J=$ $7.9 \mathrm{~Hz}, 2 \mathrm{H}), 7.53$ (d, $J=15.9 \mathrm{~Hz}, 1 \mathrm{H}), 7.42$ (q, $J=9.1,8.2$
$\mathrm{Hz}, 3 \mathrm{H}), 6.85$ (d, $J=15.9 \mathrm{~Hz}, 1 \mathrm{H}), 6.71(\mathrm{~d}, J=11.1 \mathrm{~Hz}$, 1H), 5.47 (s, 2H), 4.49 (d, $J=6.0 \mathrm{~Hz}, 2 \mathrm{H}) ;{ }^{13} \mathrm{C}$ NMR (151 MHz, DMSO) $\delta$ : $166.00,165.15,157.16(\mathrm{~d}, J=239.9 \mathrm{~Hz})$, $150.65,149.64,145.52(\mathrm{~d}, J=10.7 \mathrm{~Hz}), 143.52,136.23$, 134.44, 133.32, 131.14 (d, $J=7.2 \mathrm{~Hz}), 128.45,127.49$, $124.44(\mathrm{~d}, J=2.3 \mathrm{~Hz}), 120.96,102.74$ (d, $J=25.6 \mathrm{~Hz})$, $91.32(\mathrm{~d}, J=22.4 \mathrm{~Hz}), 42.56$; HRMS (ESI) calcd for $\mathrm{C}_{22} \mathrm{H}_{19} \mathrm{BrFN}_{4} \mathrm{O}_{2}[\mathrm{M}+\mathrm{H}]^{+}$469.0670, found 469.0672.

4.1.2 General procedure for the preparation of $7 \mathbf{a} \sim 7 \mathbf{I}$

Potassium carbonate $(0.46 \mathrm{~g}, 3.33 \mathrm{mmol})$, sodium fluoride $(47 \mathrm{mg}, 1.11 \mathrm{mmol})$, water $(5 \mathrm{~mL})$, tetrakis(triphenylphosphine) palladium (64 $\mathrm{mg}, 0.056 \mathrm{mmol})$ were added to the solvent of $6 \mathrm{a}(0.5 \mathrm{~g}, 1.11 \mathrm{mmol})$ in toluene $(20 \mathrm{~mL})$. The mixture was reaction at $100{ }^{\circ} \mathrm{C}$ under argon gas. Catalyst was filtered by diatomaceous earth and the solvent was removed under reduced pressure. The crude product was dissolved in methylene chloride and washed with water $(30 \mathrm{~mL} \times 2)$, and brine $(30 \mathrm{~mL} \times 2)$. The organic phase was dried and removed under reduced pressure. The product was purified by silica gel chromatography to give intermediate $\quad(E)-N$-(4-Amino-4'-methyl-[1,1'-biphenyl]-3yl)-4-((3-(pyridin-3-yl)acrylamido)methyl)benzamide (7a) 0.29 g, yield $56 \%$. m.p. $220 \sim 222{ }^{\circ} \mathrm{C} ;{ }^{1} \mathrm{H}$ NMR (400 MHz, DMSO- $\left.d_{6}\right) \delta: 9.72(\mathrm{~s}, 1 \mathrm{H}), 8.79(\mathrm{~s}, 2 \mathrm{H}), 8.57(\mathrm{~d}, J=$ $3.5 \mathrm{~Hz}, 1 \mathrm{H}), 8.07 \sim 7.93(\mathrm{~m}, 3 \mathrm{H}), 7.61 \sim 7.39(\mathrm{~m}, 7 \mathrm{H})$, $7.33 \sim 7.26(\mathrm{~m}, 1 \mathrm{H}), 7.20(\mathrm{~d}, J=7.9 \mathrm{~Hz}, 2 \mathrm{H}), 6.85(\mathrm{dd}, J=$ 12.0, $9.3 \mathrm{~Hz}, 2 \mathrm{H}), 5.05(\mathrm{~s}, 2 \mathrm{H}), 4.51(\mathrm{~d}, J=5.6 \mathrm{~Hz}, 2 \mathrm{H})$, $2.31(\mathrm{~s}, 3 \mathrm{H}) ;{ }^{13} \mathrm{C}$ NMR $\left(151 \mathrm{MHz}, \mathrm{DMSO}-d_{6}\right) \delta: 165.7$, $165.2,150.7,149.6,143.4,142.9,137.8,136.3,135.6$, $134.5,133.7,131.1,129.9,128.7,128.4,127.6,125.8$, $125.0,124.9,124.5,124.4,124.1,17.0,42.6,21.1$; HRMS (ESI) calcd for $\mathrm{C}_{29} \mathrm{H}_{27} \mathrm{~N}_{4} \mathrm{O}_{2}[\mathrm{M}+\mathrm{H}]^{+}$463.2129, found 463.2126 .

(E)-N-(4-Amino-[1,1'-biphenyl]-3-yl)-4-((3-(pyridin-3yl) acrylamido)methyl)benzamide (7b): Pale yellow solid, yield 53\%. m.p. $178 \sim 182{ }^{\circ} \mathrm{C}$; ${ }^{1} \mathrm{H}$ NMR $(400 \mathrm{MHz}$, DMSO- $\left.d_{6}\right) \delta: 9.72(\mathrm{~s}, 1 \mathrm{H}), 8.87 \sim 8.72(\mathrm{~m}, 2 \mathrm{H}), 8.56(\mathrm{dd}$, $J=4.7,1.4 \mathrm{~Hz}, 1 \mathrm{H}), 8.07 \sim 7.90(\mathrm{~m}, 3 \mathrm{H}), 7.58 \sim 7.50(\mathrm{~m}$, 4H), $7.48 \sim 7.42(\mathrm{~m}, 3 \mathrm{H}), 7.39(\mathrm{t}, J=7.8 \mathrm{~Hz}, 2 \mathrm{H}), 7.33$ (dd, $J=8.3,2.2 \mathrm{~Hz}, 1 \mathrm{H}), 7.24(\mathrm{t}, J=6.1 \mathrm{~Hz}, 1 \mathrm{H}), 6.90 \sim$ $6.79(\mathrm{~m}, 2 \mathrm{H}), 5.09(\mathrm{~s}, 2 \mathrm{H}), 4.51(\mathrm{~d}, J=5.9 \mathrm{~Hz}, 2 \mathrm{H}) ;{ }^{13} \mathrm{C}$ NMR (151 MHz, DMSO- $\left.d_{6}\right) \delta: 165.7,165.2,150.7,149.6$, $143.4,143.2,140.7,136.3,134.5,133.7,131.1,129.3$, $128.6,128.4,127.6,126.5,126.0,125.2,125.2,124.5$, 124.4, 124.1, 117.0, 42.6; HRMS (ESI) calcd for $\mathrm{C}_{28} \mathrm{H}_{25} \mathrm{~N}_{4} \mathrm{O}_{2} \quad[\mathrm{M}+\mathrm{H}]^{+}$449.1972, found 449.1972.

(E)- $N$-(2-Amino-5-(thiophen-2-yl)phenyl)-4-((3-(pyridin-3-yl)acrylamido)methyl)benzamide (7c): White solid, yield 33\%. m.p. $168 \sim 172{ }^{\circ} \mathrm{C} ;{ }^{1} \mathrm{H}$ NMR $(600 \mathrm{MHz}$, DMSO- $\left.d_{6}\right) \delta: 9.73(\mathrm{~s}, 1 \mathrm{H}), 8.80(\mathrm{dd}, J=12.2,3.9 \mathrm{~Hz}, 2 \mathrm{H})$, $8.57(\mathrm{dd}, J=4.7,1.4 \mathrm{~Hz}, 1 \mathrm{H}), 8.06 \sim 7.94(\mathrm{~m}, 3 \mathrm{H}), 7.54(\mathrm{~d}$, $J=15.9 \mathrm{~Hz}, 1 \mathrm{H}), 7.49(\mathrm{~d}, J=1.4 \mathrm{~Hz}, 1 \mathrm{H}), 7.48 \sim 7.42(\mathrm{~m}$, $3 \mathrm{H}), 7.36(\mathrm{dd}, J=5.0,0.8 \mathrm{~Hz}, 1 \mathrm{H}), 7.31(\mathrm{dd}, J=8.3,2.1$ $\mathrm{Hz}, 1 \mathrm{H}), 7.25$ (d, $J=2.7 \mathrm{~Hz}, 1 \mathrm{H}), 7.06(\mathrm{dd}, J=5.0,3.6 \mathrm{~Hz}$, $1 \mathrm{H}), 6.89 \sim 6.80(\mathrm{~m}, 2 \mathrm{H}), 5.16(\mathrm{~s}, 2 \mathrm{H}), 4.51(\mathrm{~d}, J=5.8 \mathrm{~Hz}$, $2 \mathrm{H}) ;{ }^{13} \mathrm{C}$ NMR (151 MHz, DMSO-d 6 ) $\delta: 165.8,165.2$, 
$150.7,149.7,144.7,143.5,143.5,136.3,134.5,133.6$, $131.1,128.7,128.4,127.5,124.5,124.4,123.9,123.7$, $122.7,121.5,116.9,42.6$; HRMS (ESI) calcd for $\mathrm{C}_{26} \mathrm{H}_{23}-$ $\mathrm{N}_{4} \mathrm{O}_{2} \mathrm{~S}[\mathrm{M}+\mathrm{H}]^{+}$455.1536, found 455.1535.

(E)-N-(2-Amino-5-(furan-2-yl)phenyl)-4-((3-(pyridin-3yl)acrylamido)methyl)benzamide (7d): Pale yellow solid, yield $37 \%$. m.p. $270 \sim 274{ }^{\circ} \mathrm{C} ;{ }^{1} \mathrm{H}$ NMR $(400 \mathrm{MHz}$, DMSO- $\left.d_{6}\right) \delta: 9.68(\mathrm{~s}, 1 \mathrm{H}), 8.83 \sim 8.73(\mathrm{~m}, 2 \mathrm{H}), 8.56(\mathrm{dd}$, $J=4.7,1.5 \mathrm{~Hz}, 1 \mathrm{H}), 8.07 \sim 7.91(\mathrm{~m}, 3 \mathrm{H}), 7.60(\mathrm{~d}, J=1.2$ $\mathrm{Hz}, 1 \mathrm{H}), 7.57 \sim 7.50(\mathrm{~m}, 2 \mathrm{H}), 7.49 \sim 7.40(\mathrm{~m}, 3 \mathrm{H}), 7.33$ $(\mathrm{dd}, \mathrm{J}=8.3,2.0 \mathrm{~Hz}, 1 \mathrm{H}), 6.87 \sim 6.77(\mathrm{~m}, 2 \mathrm{H}), 6.61(\mathrm{~d}, \mathrm{~J}=$ $3.1 \mathrm{~Hz}, 1 \mathrm{H}), 6.50(\mathrm{dd}, J=3.3,1.8 \mathrm{~Hz}, 1 \mathrm{H}), 5.14(\mathrm{~s}, 2 \mathrm{H})$, $4.50(\mathrm{~d}, J=5.9 \mathrm{~Hz}, 2 \mathrm{H}) ;{ }^{13} \mathrm{C}$ NMR $\left(151 \mathrm{MHz}, \mathrm{DMSO}-d_{6}\right)$ $\delta: 165.8,165.2,154.3,150.7,149.6,143.4,141.7,136.3$, $134.5,133.6,131.1,128.4,127.6,124.5,124.4,123.7$, 122.8, 119.7, 116.7, 112.3, 102.9, 42.6; HRMS (ESI) calcd for $\mathrm{C}_{26} \mathrm{H}_{23} \mathrm{~N}_{4} \mathrm{O}_{3} \quad[\mathrm{M}+\mathrm{H}]^{+}$439.1765, found 439.1765.

(E)-N-(4-Amino-4'-fluoro-[1,1'-biphenyl]-3-yl)-4-((3(pyridin-3-yl)acrylamido)methyl)benzamide (7e): White solid, yield 36\%. m.p. 212 214 ${ }^{\circ} \mathrm{C}$; ${ }^{1} \mathrm{H}$ NMR $(600 \mathrm{MHz}$, DMSO- $\left.d_{6}\right) \delta: 9.74(\mathrm{~s}, 1 \mathrm{H}), 8.82(\mathrm{t}, J=5.9 \mathrm{~Hz}, 1 \mathrm{H}), 8.79(\mathrm{~d}$, $J=2.1 \mathrm{~Hz}, 1 \mathrm{H}), 8.56(\mathrm{dd}, J=4.8,1.5 \mathrm{~Hz}, 1 \mathrm{H}), 8.05 \sim 8.00$ (m, 1H), 7.99 (d, $J=8.1 \mathrm{~Hz}, 2 \mathrm{H}), 7.58(\mathrm{dt}, J=10.7,5.4$ $\mathrm{Hz}, 2 \mathrm{H}), 7.54$ (d, $J=15.9 \mathrm{~Hz}, 1 \mathrm{H}), 7.51(\mathrm{~d}, J=1.4 \mathrm{~Hz}$, $1 \mathrm{H}), 7.47 \sim 7.42(\mathrm{~m}, 3 \mathrm{H}), 7.30(\mathrm{dd}, J=8.3,2.2 \mathrm{~Hz}, 1 \mathrm{H})$, $7.24 \sim 7.18(\mathrm{~m}, 2 \mathrm{H}), 6.85(\mathrm{dt}, J=15.4,8.2 \mathrm{~Hz}, 2 \mathrm{H}), 5.11$ $(\mathrm{s}, 2 \mathrm{H}), 4.51(\mathrm{~d}, J=5.7 \mathrm{~Hz}, 2 \mathrm{H}) ;{ }^{13} \mathrm{C} \mathrm{NMR}(151 \mathrm{MHz}$, DMSO- $\left.d_{6}\right) \delta: 165.7,165.2,161.5(\mathrm{~d}, J=242.7 \mathrm{~Hz}), 150.7$, 149.7, 143.4, 143.2, 137.2 (d, $J=3.2 \mathrm{~Hz}), 136.3,134.5$, $133.7,131.1,128.4,127.8$ (d, $J=8.1 \mathrm{~Hz}), 127.6,127.6$, $125.3,125.1,124.5,124.4,124.1,117.0,116.0(\mathrm{~d}, J=21.3$ $\mathrm{Hz}$ ), 42.6; HRMS (ESI) calcd for $\mathrm{C}_{28} \mathrm{H}_{24} \mathrm{FN}_{4} \mathrm{O}_{2}[\mathrm{M}+\mathrm{H}]^{+}$ 467.1878 , found 467.1876

(E)-N-(2-Amino-5-(pyridin-4-yl)phenyl)-4-((3-(pyridin3-yl)acrylamido)methyl)benzamide (7f): Tan solid, yield 28\%. m.p. 98 $100{ }^{\circ} \mathrm{C} ;{ }^{1} \mathrm{H}$ NMR (600 MHz, DMSO- $\left.d_{6}\right) \delta$ : $9.76(\mathrm{~s}, 1 \mathrm{H}), 8.83$ (t, $J=5.8 \mathrm{~Hz}, 1 \mathrm{H}), 8.79(\mathrm{~s}, 1 \mathrm{H}), 8.57$ (d, $J=3.8 \mathrm{~Hz}, 1 \mathrm{H}), 8.52(\mathrm{~d}, J=5.7 \mathrm{~Hz}, 2 \mathrm{H}), 8.05 \sim 7.96(\mathrm{~m}$, $3 \mathrm{H}), 7.70(\mathrm{~s}, 1 \mathrm{H}), 7.59(\mathrm{~d}, J=5.6 \mathrm{~Hz}, 2 \mathrm{H}), 7.56 \sim 7.49(\mathrm{~m}$, $2 \mathrm{H}), 7.48 \sim 7.42(\mathrm{~m}, 3 \mathrm{H}), 6.90(\mathrm{~d}, J=8.4 \mathrm{~Hz}, 1 \mathrm{H}), 6.85(\mathrm{~d}$, $J=15.9 \mathrm{~Hz}, 1 \mathrm{H}), 5.39(\mathrm{~s}, 2 \mathrm{H}), 4.51(\mathrm{~d}, J=5.7 \mathrm{~Hz}, 2 \mathrm{H}) ;{ }^{13} \mathrm{C}$ NMR (151 MHz, DMSO- $\left.d_{6}\right) \delta$ : 165.9, 165.2, 150.7, 150.5, $149.7,147.2,145.3,143.5,136.3,134.5,133.6,131.1$, $128.5,127.5,125.7,125.5,124.6,124.5,124.4,123.9$, 120.1, 116.7, 42.6; HRMS (ESI) calcd for $\mathrm{C}_{27} \mathrm{H}_{24} \mathrm{~N}_{5} \mathrm{O}_{2}$ $[\mathrm{M}+\mathrm{H}]^{+}$450.1925, found 450.1930.

(E)- $N$-(2-Amino-5-(furan-3-yl)phenyl)-4-((3-(pyridin-3yl)acrylamido)methyl)benzamide (7g): Light grey solid, yield $21 \%$. m.p. $188 \sim 192{ }^{\circ} \mathrm{C} ;{ }^{1} \mathrm{H}$ NMR $(600 \mathrm{MHz}$, DMSO- $\left.d_{6}\right) \delta: 9.74(\mathrm{~s}, 1 \mathrm{H}), 8.82(\mathrm{t}, J=5.9 \mathrm{~Hz}, 1 \mathrm{H}), 8.79$ (d, $J=2.0 \mathrm{~Hz}, 1 \mathrm{H}), 8.57$ (dd, $J=4.7,1.5 \mathrm{~Hz}, 1 \mathrm{H}), 8.04 \sim 7.95$ $(\mathrm{m}, 4 \mathrm{H}), 7.67(\mathrm{t}, J=1.6 \mathrm{~Hz}, 1 \mathrm{H}), 7.55(\mathrm{~d}, J=16.2 \mathrm{~Hz}, 1 \mathrm{H})$, $7.48 \sim 7.42(\mathrm{~m}, 3 \mathrm{H}), 7.41(\mathrm{~d}, J=1.4 \mathrm{~Hz}, 1 \mathrm{H}), 7.25(\mathrm{dd}, J=$ 8.2, $2.0 \mathrm{~Hz}, 1 \mathrm{H}), 6.87 \sim 6.79(\mathrm{~m}, 3 \mathrm{H}), 4.99(\mathrm{~s}, 2 \mathrm{H}), 4.51(\mathrm{~d}$, $J=5.7 \mathrm{~Hz}, 2 \mathrm{H}) ;{ }^{13} \mathrm{C}$ NMR $\left(151 \mathrm{MHz}, \mathrm{DMSO}-d_{6}\right) \delta: 165.6$, $165.2,150.7,149.7,144.3,143.4,142.9,137.8,136.3$, $134.5,133.6,131.1,128.4,127.5,126.4,124.7,124.6$,
$124.5,124.4,124.0,120.7,116.8,109.0$, 42.6; HRMS (ESI) calcd for $\mathrm{C}_{26} \mathrm{H}_{23} \mathrm{~N}_{4} \mathrm{O}_{3}[\mathrm{M}+\mathrm{H}]^{+}$439.439.1765, found 439.1766 .

(E)-N-(4-Amino-6-fluoro-4'-methyl-[1,1'-biphenyl]-3yl)-4-((3-(pyridin-3-yl)acrylamido)methyl)benzamide (7h): Reddish brown solid, yield 31\%. m.p. 106 108 ${ }^{\circ} \mathrm{C} ;{ }^{1} \mathrm{H}$ NMR (400 MHz, DMSO- $\left.d_{6}\right) \delta: 9.67(\mathrm{~s}, 1 \mathrm{H}), 8.80(\mathrm{~s}, 2 \mathrm{H})$, 8.57 (d, $J=3.9 \mathrm{~Hz}, 1 \mathrm{H}), 8.00$ (t, $J=8.3 \mathrm{~Hz}, 3 \mathrm{H}), 7.55$ (d, $J=15.9 \mathrm{~Hz}, 1 \mathrm{H}), 7.51 \sim 7.40(\mathrm{~m}, 3 \mathrm{H}), 7.36(\mathrm{~d}, J=7.4 \mathrm{~Hz}$, 2H), $7.31(\mathrm{~d}, J=8.5 \mathrm{~Hz}, 1 \mathrm{H}), 7.22$ (d, $J=7.9 \mathrm{~Hz}, 2 \mathrm{H}), 6.85$ $(\mathrm{d}, J=15.9 \mathrm{~Hz}, 1 \mathrm{H}), 6.66(\mathrm{~d}, J=13.1 \mathrm{~Hz}, 1 \mathrm{H}), 5.35$ (s, $2 \mathrm{H}), 4.51(\mathrm{~d}, J=5.7 \mathrm{~Hz}, 2 \mathrm{H}), 2.32(\mathrm{~s}, 3 \mathrm{H}) ;{ }^{13} \mathrm{C} \mathrm{NMR}(151$ MHz, DMSO- $\left.d_{6}\right) \delta: 165.9,165.2,158.3(\mathrm{~d}, J=242.2 \mathrm{~Hz}$,), $150.7,149.7,145.1$ (d, $J=11.9 \mathrm{~Hz}), 143.4,136.3,136.2$, $134.5,133.6133 .2,131.1,129.6,128.7$ (d, $J=5.7 \mathrm{~Hz})$, $128.4,128.4,127.5,124.5,124.4,120.2(\mathrm{~d}, J=1.7 \mathrm{~Hz})$, 115.7 (d, $J=14.6 \mathrm{~Hz}), 102.7$ (d, $J=26.8 \mathrm{~Hz}), 42.6,21.2$; HRMS (ESI) calcd for $\mathrm{C}_{29} \mathrm{H}_{26} \mathrm{FN}_{4} \mathrm{O}_{2}[\mathrm{M}+\mathrm{H}]^{+}$481.2034, found 481.2034 .

(E)-N-(4-Amino-6-fluoro-[1,1'-biphenyl]-3-yl)-4-((3(pyridin-3-yl)acrylamido)methyl)benzamide (7i): Pale yellow solid, yield $41 \%$. m.p. $220 \sim 222{ }^{\circ} \mathrm{C} ;{ }^{1} \mathrm{H}$ NMR (400 MHz, DMSO- $\left.d_{6}\right) \delta: 9.65(\mathrm{~s}, 1 \mathrm{H}), 8.82 \sim 8.74(\mathrm{~m}, 2 \mathrm{H}), 8.56$ $(\mathrm{dd}, \mathrm{J}=4.7,1.5 \mathrm{~Hz}, 1 \mathrm{H}), 8.03 \sim 7.94(\mathrm{~m}, 3 \mathrm{H}), 7.53(\mathrm{~d}, J=$ $15.9 \mathrm{~Hz}, 1 \mathrm{H}), 7.48 \sim 7.38(\mathrm{~m}, 7 \mathrm{H}), 7.34 \sim 7.24(\mathrm{~m}, 2 \mathrm{H})$, $6.83(\mathrm{~d}, J=15.9 \mathrm{~Hz}, 1 \mathrm{H}), 6.65(\mathrm{~d}, J=13.1 \mathrm{~Hz}, 1 \mathrm{H}), 5.38$ $(\mathrm{s}, 2 \mathrm{H}), 4.50(\mathrm{~d}, J=5.9 \mathrm{~Hz}, 2 \mathrm{H}) ;{ }^{13} \mathrm{C} \mathrm{NMR}(151 \mathrm{MHz}$, DMSO- $\left.d_{6}\right) \delta: 166.0,165.2,158.2(\mathrm{~d}, J=243.6 \mathrm{~Hz}), 150.7$, $149.6,145.3(\mathrm{~d}, J=12.0 \mathrm{~Hz}) 143.4,136.3,134.5,133.6$, $131.1,129.1$ (d, $J=1.4 \mathrm{~Hz}), 129.0,128.6,128.5,128.4$, $127.5,127.0,124.5,124.4,120.2$ (d, $J=2.1 \mathrm{~Hz}), 115.7$ (d, $J=14.2 \mathrm{~Hz}$ ), $102.8(\mathrm{~d}, J=26.9 \mathrm{~Hz}), 42.6$; HRMS (ESI) calcd for $\mathrm{C}_{28} \mathrm{H}_{24} \mathrm{FN}_{4} \mathrm{O}_{2}[\mathrm{M}+\mathrm{H}]{ }^{+}$467.1878, found 467.1874 .

(E)-N-(2-Amino-4-fluoro-5-(thiophen-2-yl)phenyl)-4((3-(pyridin-3-yl)acrylamido)methyl)benzamide (7j): Yellow solid, yield $37 \%$. m.p. $134 \sim 138{ }^{\circ} \mathrm{C} ;{ }^{1} \mathrm{H}$ NMR $(400$ $\left.\mathrm{MHz}, \mathrm{DMSO}-d_{6}\right) \delta$ : $9.69(\mathrm{~s}, 1 \mathrm{H}), 8.82(\mathrm{t}, J=6.0 \mathrm{~Hz}, 1 \mathrm{H})$, $8.78(\mathrm{~d}, J=1.6 \mathrm{~Hz}, 1 \mathrm{H}), 8.56(\mathrm{dd}, J=4.7,1.3 \mathrm{~Hz}, 1 \mathrm{H})$, $8.04 \sim 7.92(\mathrm{~m}, 3 \mathrm{H}), 7.57 \sim 7.38(\mathrm{~m}, 6 \mathrm{H}), 7.29(\mathrm{~d}, J=3.6$ $\mathrm{Hz}, 1 \mathrm{H}), 7.12 \sim 7.06(\mathrm{~m}, 1 \mathrm{H}), 6.84(\mathrm{~d}, J=15.6,1 \mathrm{H}), 6.65$ $(\mathrm{d}, J=13.4 \mathrm{~Hz}, 1 \mathrm{H}), 5.48(\mathrm{~s}, 2 \mathrm{H}), 4.50(\mathrm{~d}, J=5.9 \mathrm{~Hz}, 2 \mathrm{H})$; ${ }^{13} \mathrm{C}$ NMR (151 MHz, DMSO- $\left.d_{6}\right) \delta: 166.0,165.2,157.5(\mathrm{~d}$, $J=244.0 \mathrm{~Hz}), 150.7,149.6,145.4(\mathrm{~d}, J=12.0 \mathrm{~Hz}), 143.5$, $137.6(\mathrm{~d}, J=3.9 \mathrm{~Hz}), 136.3,134.5,133.5,131.1,128.5$, 128.2, 127.5, 127.3 (d, $J=5.6 \mathrm{~Hz}), 124.8$ (d, $J=4.2 \mathrm{~Hz})$, 124.5, 124.4, 124.2 (d, $J=4.9 \mathrm{~Hz}), 120.2,109.3$ (d, $J=$ $14.5 \mathrm{~Hz}), 102.4$ (d, $J=25.8 \mathrm{~Hz}$ ), 42.6; HRMS (ESI) calcd for $\mathrm{C}_{26} \mathrm{H}_{22} \mathrm{FN}_{4} \mathrm{O}_{2} \mathrm{~S}[\mathrm{M}+\mathrm{H}]^{+}$473.1442, found 473.1437 .

(E)- $N$-(2-Amino-4-fluoro-5-(pyridin-4-yl)phenyl)-4-((3(pyridin-3-yl)acrylamido)methyl)benzamide (7k): White solid, yield 36\%. m.p. 202 204 ${ }^{\circ} \mathrm{C} ;{ }^{1} \mathrm{H}$ NMR (600 MHz, DMSO-d $\left.d_{6}\right) \delta: 9.70(\mathrm{~s}, 1 \mathrm{H}), 8.87 \sim 8.74(\mathrm{~m}, 2 \mathrm{H}), 8.61 \sim$ $8.50(\mathrm{~m}, 3 \mathrm{H}), 8.05 \sim 7.92(\mathrm{~m}, 3 \mathrm{H}), 7.57 \sim 7.52(\mathrm{~m}, 1 \mathrm{H})$, $7.51 \sim 7.41(\mathrm{~m}, 6 \mathrm{H}), 6.84(\mathrm{~d}, J=13.6 \mathrm{~Hz}, 1 \mathrm{H}), 6.67(\mathrm{~d}, J=$ $13.6 \mathrm{~Hz}, 1 \mathrm{H}), 5.67(\mathrm{~s}, 2 \mathrm{H}), 4.51(\mathrm{~d}, J=5.9 \mathrm{~Hz}, 2 \mathrm{H}) ;{ }^{13} \mathrm{C}$ NMR (151 MHz, DMSO- $\left.d_{6}\right) \delta: 166.1,165.2,158.9(\mathrm{~d}, J=$ 
$245.1 \mathrm{~Hz}), 150.7,150.3,149.7,147.1$ (d, $J=12.5 \mathrm{~Hz})$, $143.4,136.3,134.5,133.6,131.1,128.9$ (d, $J=5.4 \mathrm{~Hz})$, $128.5,127.5,124.5,124.4,122.8,122.7,120.4,112.0(\mathrm{~d}$, $J=13.3 \mathrm{~Hz}$ ), 102.3 (d, $J=26.5 \mathrm{~Hz}), 42.6$; HRMS (ESI) calcd for $\mathrm{C}_{27} \mathrm{H}_{23} \mathrm{FN}_{5} \mathrm{O}_{2}[\mathrm{M}+\mathrm{H}]^{+}$468.1830, found 468.1828 .

(E)-N-(2-Amino-4-fluoro-5-(furan-3-yl)phenyl)-4-((3(pyridin-3-yl)acrylamido)methyl)benzamide (7l): Grayish-brown solid, yield 19\%. m.p. 106 108 ${ }^{\circ} \mathrm{C} ;{ }^{1} \mathrm{H}$ NMR $\left(600 \mathrm{MHz}, \mathrm{DMSO}-d_{6}\right) \delta: 9.69(\mathrm{~s}, 1 \mathrm{H}), 8.82(\mathrm{t}, J=6.0 \mathrm{~Hz}$, $1 \mathrm{H}), 8.79(\mathrm{~d}, J=2.1 \mathrm{~Hz}, 1 \mathrm{H}), 8.57(\mathrm{dd}, J=4.8,1.6 \mathrm{~Hz}$, $1 \mathrm{H}), 8.04 \sim 8.00(\mathrm{~m}, 1 \mathrm{H}), 7.99(\mathrm{~d}, J=8.2 \mathrm{~Hz}, 2 \mathrm{H}), 7.90$ (s, $1 \mathrm{H}), 7.71(\mathrm{t}, J=1.7 \mathrm{~Hz}, 1 \mathrm{H}), 7.54(\mathrm{~d}, J=12.0 \mathrm{~Hz}, 1 \mathrm{H})$, $7.48 \sim 7.40(\mathrm{~m}, 4 \mathrm{H}), 6.87 \sim 6.82(\mathrm{~m}, 2 \mathrm{H}), 6.64(\mathrm{~d}, J=13.1$ $\mathrm{Hz}, 1 \mathrm{H}), 5.31$ (s, 2H), $4.51(\mathrm{~d}, J=6.0 \mathrm{~Hz}, 2 \mathrm{H}) ;{ }^{13} \mathrm{C} \mathrm{NMR}$ $\left(151 \mathrm{MHz}, \mathrm{DMSO}-d_{6}\right) \delta$ : $165.9,165.2,158.5$ (d, $J=243.6$ $\mathrm{Hz}), 150.7,149.6,144.9$ (d, $J=12.2 \mathrm{~Hz}), 143.9,143.4$, $139.6(\mathrm{~d}, J=11.0 \mathrm{~Hz}), 136.3,134.5,133.5,131.1,128.4$, $127.5,127.3(\mathrm{~d}, J=7.0 \mathrm{~Hz}), 124.5,124.4,120.4(\mathrm{~d}, J=2.3$ $\mathrm{Hz}), 120.0,109.4,107.2$ (d, $J=15.8 \mathrm{~Hz}), 102.46$ (d, $J=$ $26.1 \mathrm{~Hz}$ ), 42.6; HRMS (ESI) calcd for $\mathrm{C}_{26} \mathrm{H}_{22} \mathrm{FN}_{4} \mathrm{O}_{3}[\mathrm{M}+$ $\mathrm{H}]^{+}$457.1670, found 457.1672.

\subsection{Biological activity}

\subsubsection{In vitro HDACs inhibition fluorescence assay}

In vitro HDAC inhibition assays were conducted as previously described. ${ }^{[16]}$ First, HeLa nuclear extract (mainly containing HDAC1 and HDAC2) was used to be the enzyme source, and all the target compounds were preliminarily screened for HDACs inhibition activity. Blank group, 100\% control group, compound group and positive drug group were set up in the experiment. Each group was parallel to three times. $10 \mu \mathrm{L}$ of enzyme solution (HeLa nuclear extracts) was mixed with various concentrations of target compounds and reference compounds Chidamide and MS275 $(50 \mu \mathrm{L})$, with $100 \%$ and none HDACs groups being control group. The mixture were then incubated at $37{ }^{\circ} \mathrm{C}$ for $5 \mathrm{~min}$, followed by adding $40 \mu \mathrm{L}$ of fluorogenic substrate (Boc-Lys (Ac)-AMC for HeLa nuclear extracts; After incubation at $37{ }^{\circ} \mathrm{C}$ for $30 \mathrm{~min}$, the mixture was quenched by addition of $100 \mu \mathrm{L}$ of developer containing trypsin and TSA. Over another incubation at $37{ }^{\circ} \mathrm{C}$ for 20 min, fluorescence intensity was measured by a microplate reader at excitation and emission wavelengths of 390 and $460 \mathrm{nmol} / \mathrm{L}$, respectively. The inhibition ratios were calculated from the fluorescence intensity readings of tested wells relative to those of control wells, and the $\mathrm{IC}_{50}$ values were calculated using a regression analysis of the concentration inhibition data.
4.2.2 In vitro anti-tumor cell proliferation assay

In vitro antiproliferative assays were determined by the MTT (3-[4,5-dimethyl-2-thiazolyl]-2,5-diphenyl-2h-tetrazolium bromide) method as previously described. ${ }^{[17]}$ In general, all cell lines were preserved in liquid nitrogen and recovered before use. All the cell lines were maintained in RPMI1640 medium containing 10\% FBS at $37{ }^{\circ} \mathrm{C}$ in $5 \%$ $\mathrm{CO}_{2}$ humidified incubator. Cells were passaged the day before dosing into a 96-well plate, allowed to grow for $4 \mathrm{~h}$, and then treated with different concentrations of tested compound. The plated were incubated for an additional 48 $\mathrm{h}$ afterwards, and then a $0.5 \%$ MTT solution was added to each well. After incubation for another $4 \mathrm{~h}$, formazan formed from MTT was extracted by addition of $150 \mu \mathrm{L}$ of DMSO and the plate was rocked for $15 \mathrm{~min}$. Absorbance was then determined using an ELISA reader at $570 \mathrm{~nm}$.

Supporting Information ${ }^{1} \mathrm{H}$ NMR and ${ }^{13} \mathrm{C}$ NMR spectra of compounds $\mathbf{7 a} \sim \mathbf{7 l}$ and intermediates $\mathbf{3 b}, \mathbf{4 b}, \mathbf{6 b}$. The Supporting Information is available free of charge via the Internet at http://siocjournal.cn/.

\section{References}

[1] Khorasanizadeh, S. Cell 2004, 116, 259.

[2] Kouzarides, T. Cell 2007, 128, 693.

[3] Brownell, J. E.; Zhou, J.; Ranalli, T.; Kobayashi, R.; Edmondson, D. G.; Roth, S. Y.; Allis, C. D. Cell 1996, 84, 843.

[4] Saha, R. N.; Pahan, K. Cell Death Differ. 2005, 13, 539.

[5] Zhang, J.; Zhong, Q. Cell. Mol. Life Sci. 2014, 71, 3885.

[6] Omar, K.; Thangue, N. B. L. Immunol. Cell Biol. 2012, 90, 85.

[7] Qiao, Z.; Ren, S.; Li, W.; Wang, X.; He, M.; Guo, Y.; Sun, L.; He, Y.; Ge, Y.; Yu, Q. Biochem. Biophys. Res. Commun. 2013, 434, 95.

[8] Ning, Z. Q.; Li, Z. B.; Newman, M. J.; Shan, S.; Wang, X. H.; Pan, D. S.; Zhang, J.; Dong, M.; Du, X.; Lu, X. P. Cancer Chemother. Pharmacol. 2012, 69, 901.

[9] Zhou, J.; Xie, H.; Liu, Z.; Luo, H. B.; Wu, R. J. Chem. Inf. Model. 2014, 54, 3162.

[10] Hu, X. S.; Wang, L.; Lin, L.; Han, X. H.; Dou, G. F.; Meng, Z. Y.; Shi, Y. K. Chin. J. Cancer Res. 2016, $28,444$.

[11] Jiao, J.; Hu, J. Q.; Fang, H. Chin. J. New Drug 2010, 386 (in Chinese). (焦杰，胡建强，方浩，中国新药杂志, 2010, 386.)

[12] Zhu, H. F.; Tong, H.; Gong, Y. Y.; Shao, S. Y.; Deng, C. M.; Yuan, W. Z.; Zhang, Y. M. J. Polym. Sci., Part A: Polym. Chem. 2012, 50, 2172 .

[13] Zhao, W. N.; Ghosh, B.; Tyler, M.; Lalonde, J.; Joseph, N. F.; Kosaric, N.; Fass, D. M.; Tsai, L. H.; Mazitschek, R.; Haggarty, S. J. ACS Chem. Neurosci. 2018, 9, 2262.

[14] Yoon, H. J.; Yang, S. J.; Gong, Y. D. ACS Comb. Sci. 2017, 19, 738.

[15] Hornberger, K. R.; Badiang, J. G.; Salovich, J. M.; Kuntz, K. W.; Emmitte, K. A.; Cheung, M. Tetrahedron Lett. 2008, 49, 6348.

[16] Li, X. Y.; Inks, E. S.; Li, X. G; Hou, J. N.; Chou, C. J.; Zhang, J.; Jiang, Y. Q.; Zhang, Y. J.; Xu, W. F. J. Med. Chem. 2014, 57, 3324.

[17] Yu, C. G.; He, F.; Qu, Y.; Zhang, Q. Q.; Lv, J. H.; Zhang, X. N.; Xu, A. N.; Miao, P. N.; Wu, J. D. Bioorg. Med. Chem. 2018, 26, 1859.

(Zhao, X.) 